\title{
Occupation times of exclusion processes
}

\author{
Patrícia Gonçalves
}

\begin{abstract}
In this paper we consider exclusion processes $\left\{\eta_{t}: t \geq 0\right\}$ evolving on the one-dimensional lattice $\mathbb{Z}$, under the diffusive time scale $t n^{2}$ and starting from the invariant state $\nu_{\rho}$ - the Bernoulli product measure of parameter $\rho \in[0,1]$. Our goal consists in establishing the scaling limits of the additive functional $\Gamma_{t}:=\int_{0}^{t n^{2}} \eta_{s}(0) d s$ - the occupation time of the origin. We present a method, recently introduced in [7], from which a local Boltzmann-Gibbs Principle can be derived for a general class of exclusion processes. In this case, this principle says that $\Gamma_{t}$ is very well approximated to the additive functional of the density of particles. As a consequence, the scaling limits of $\Gamma_{t}$ follow from the scaling limits of the density of particles. As examples we present the mean-zero exclusion, the symmetric simple exclusion and the weakly asymmetric simple exclusion. For the latter under a strong asymmetry regime, the limit of $\Gamma_{t}$ is given in terms of the solution of the KPZ equation.
\end{abstract}

\section{Introduction}

In these notes we will explore the answer to the following question: given a one-dimensional Markov interaction $\left\{\eta_{t}: t \geq 0\right\}$ with state space $\Omega$ and a function $f: \Omega \rightarrow \mathbb{R}$, what is the scaling limit of the additive functional:

$$
\Gamma_{t}(f):=\int_{0}^{t} f\left(\eta_{s}\right) d s
$$

There is a vast literature on the study of scaling limits of additive functionals of particles systems, but we point out here the seminal work [10], in

Patrícia Gonçalves

Centro de Matemática da Universidade do Minho, Campus de Gualtar, 4710-057 Braga, Portugal e-mail: patg@math.uminho.pt 
which the authors give a characterization of the functions $f$, for which $\Gamma_{t}$ has a Brownian motion as scaling limit. There, the study of $\Gamma_{t}$ was motivated by the analysis of the motion of a tagged particle. The relation is that, the motion of the tagged particle can be written as a martingale plus an additive functional. Standard theorems for martingales provide the limit of the martingale term, so it remains to characterize the limit of the additive functional, in order to determine the scaling limits of the tagged particle. In [10] they give an abstract condition on $f$ under which the additive functional converges. This condition is described as follows. Suppose that $\left\{\eta_{t}: t \geq 0\right\}$ is a stationary Markov process with state space $\mathcal{E}$, with generator $\mathcal{L}$ and that it is reversible with respect to some probability measure $\nu$. For a function $f$ such that $E_{\nu}[f(\eta)]=0, \theta^{-1 / 2} \Gamma_{\theta t}(f)$ converges to a Brownian motion, as long as, $\lim _{t \rightarrow \infty} t^{-1} E_{\nu}\left[\left(\Gamma_{t}(f)\right)^{2}\right]<\infty[10]$.

By exploring $t^{-1} E_{\nu}\left[\left(\Gamma_{t}(f)\right)^{2}\right]$, last condition is equivalent to requiring that $f \in \mathcal{H}^{-1}$. The Sobolev space $\mathcal{H}^{-1}$ is the dual, with respect to $L^{2}(\nu)$, of the space $\mathcal{H}^{1}$, defined as the set of functions $f \in L^{2}(\nu)$ such that $\|f\|_{1}^{2}=$ $\int_{\varepsilon}-f(\eta)(\Omega f)(\eta) \nu(d \eta)<\infty$. Usually, is not easy to verify that $f \in \mathcal{H}^{-1}$ and in [15] they came out with a very simple criterium, which gives the Brownian motion limit of $\Gamma_{t}(f)$. Following the terminology of [15], a local function $f: \mathcal{E} \rightarrow \mathbb{R}$ whose limiting variance $t^{-1} E_{\nu}\left[\left(\Gamma_{t}(f)\right)^{2}\right]$ is finite (or equivalently $\left.f \in \mathcal{H}^{-1}\right)$ is called an admissible function. In that paper the authors prove that for the one-dimensional finite range symmetric exclusion, a function $f$ is admissible for the generator acting on $L^{2}\left(\mathbb{P}_{\rho}\right)$ (where $\mathbb{P}_{\rho}$ is the distribution of the exclusion process starting from $\nu_{\rho}$ ), if and only if, defining for $\rho \in(0,1)$ $\varphi_{f}(\rho):=E_{\nu_{\rho}}[f(\eta)]$, we have that

$$
\left.\varphi_{f}^{j}(\tilde{\rho})\right|_{\tilde{\rho}=\rho}=0, \quad \text { for } \quad j=0,1,2 .
$$

This condition is equivalent to saying that the degree of $f$ is greater or equal to three, namely: $f$ can be written as $f(\eta):=c \prod_{x \in A}(\eta(x)-\rho)$, where $c$ is a constant and $A \subseteq \mathbb{Z}$ with $|A| \geq 3$. In that paper the same result is proved for one-dimensional finite range symmetric zero-range processes, under the condition that, the inverse of the spectral gap for the dynamics restricted to a finite box of size $\ell$ has second moment bounded from above by $c \ell^{4}$, where $c$ is a constant.

In $[6,7,8]$ we came across with the study of additive functionals, when establishing the equilibrium fluctuations of exclusion type models. In those papers, we establish the limit process governing the fluctuations of particle systems of exclusion type and when characterizing this process as the solution of some stochastic partial differential equation we had the need to derive the Boltzmann-Gibbs Principle. This principle was introduced in [2] and says that:

$$
\int_{0}^{t} \frac{1}{\sqrt{n}} \sum_{x \in \mathbb{Z}} g(x / n)\left\{\tau_{x} f\left(\eta_{s}\right)-\varphi_{f}(\rho)-\varphi_{f}^{\prime}(\rho)\left(\eta_{s}(x)-\rho\right) d s\right\} \underset{n \rightarrow+\infty}{\longrightarrow} 0,
$$


in $L^{2}\left(\mathbb{P}_{\rho}\right)$. Here $g$ is a test function sufficiently smooth and $f$ is a local function defined on $\mathcal{E}$ - the state space of the Markov process $\left\{\eta_{t}: t \geq 0\right\}$. In $[6,7,8]$ we establish a stronger Boltzmann-Gibbs Principle under which, we can identify the limit of the functional above by speeding the processes into longer time scales/stronger asymmetries. Contrarily to our initial purposes, in the previous additive functional, the integrand function is no longer local - since it depends on the process defined on the full lattice. Nevertheless, the proof of the stronger Boltzmann-Gibbs Principle derived in $[6,7,8]$ can be formulated in terms of local functions and in [9] we derived the local Boltzmann-Gibbs Principle, for exclusion processes satisfying the conditions of section 2 :

Theorem 1 (Local Boltzmann-Gibbs Principle [9]).

Let $f: \mathcal{E} \rightarrow \mathbb{E}$ be a local function, such that $\operatorname{supp}(f) \subseteq\{1, \ldots, k\}$ and $\varphi_{f}(\rho)=0$. There exists $c=c(f, \rho)$ such that

i) if $\varphi_{f}^{\prime}(\rho) \neq 0$, then for any $t \geq 0$ and any $\ell \geq k$ :

$$
\mathbb{E}_{\rho}\left[\left(\int_{0}^{t}\left\{f\left(\eta_{s}\right)-\varphi_{f}^{\prime}(\rho)\left(\eta_{s}^{\ell}-\rho\right)\right\} d s\right)^{2}\right] \leq c\left(t \ell+\frac{t^{2}}{\ell^{2}}\right)
$$

ii) if $\varphi_{f}^{\prime}(\rho)=0$, then for any $t \geq 0$ and any $\ell \geq k$ :

$$
\mathbb{E}_{\rho}\left[\left(\int_{0}^{t}\left\{f\left(\eta_{s}\right)-\frac{\varphi_{f}^{\prime \prime}(\rho)}{2}\left(\left(\eta_{s}^{\ell}-\rho\right)^{2}-\frac{\rho(1-\rho)}{\ell}\right)\right\} d s\right)^{2}\right] \leq c\left(t(\log \ell)^{2}+\frac{t^{2}}{\ell^{3}}\right)
$$

where $\eta^{\ell}:=\frac{1}{\ell} \sum_{x=1}^{\ell} \eta(x)$ and $\mathbb{E}_{\rho}$ denotes the expectation with respect to $\mathbb{P}_{\rho}$.

We will see below that last result allow us to obtain upper bounds on the variance of additive functionals for local functions such that $\varphi_{f}(\rho)=0$ and $\varphi_{f}^{\prime}(\rho) \neq 0$ or such that $\varphi_{f}(\rho)=\varphi_{f}^{\prime}(\rho)=0$ and $\varphi_{f}^{\prime \prime}(\rho) \neq 0$. Notice that these functions do not satisfy the admissibility condition (1) as stated in [15]. Moreover, in the case $\varphi_{f}^{\prime}(\rho) \neq 0$, we can also identify the limit of $\Gamma_{t}(f)$ as a fractional Brownian motion of Hurst exponent $H=3 / 4$, for a general class of exclusion processes.

We recall from [15], that in the symmetric finite range exclusion, for an admissible function $f: \mathcal{E} \rightarrow \mathbb{R}$ as in (1) the variance of $\Gamma_{t}(f)$ is bounded from above by $C t$ and the invariance principle for $\Gamma_{t}(f)$ was also established:

$$
\frac{1}{\sqrt{\theta}} \int_{0}^{\theta t} f\left(\eta_{s}\right) d s \underset{\theta \rightarrow+\infty}{\longrightarrow} \mathcal{B}(C t)
$$

where $\mathcal{B}$ is the standard Brownian motion and $C$ is a constant. In that paper, (2) is also proved for the case of symmetric zero-range processes, under the condition on the spectral gap mentioned above. In $[13,16]$, the previous result was obtained for mean-zero symmetric exclusion processes. In [13] it is also 
proved that for mean-zero simple exclusion processes, the set of admissible functions are those satisfying condition (1). In the finite range symmetric and mean-zero exclusion process, for a local function $f$ such that $\varphi_{f}(\rho)=0$ and $\varphi_{f}^{\prime}(\rho) \neq 0$, the variance of $\Gamma_{t}(f)$ is bounded from above by $C t^{3 / 2}$ and

$$
\frac{1}{\theta^{3 / 4}} \int_{0}^{\theta t} f\left(\eta_{s}\right) d s \underset{\theta \rightarrow+\infty}{\longrightarrow} \mathcal{B}_{3 / 4}(C t),
$$

where $\mathcal{B}_{3 / 4}$ is the fractional Brownian motion with Hurst exponent $H=3 / 4$ and $C$ is a constant [13]. For symmetric zero-range processes the limit (3) was established in [12].

It remains to cover the case of local functions $f$ such that $\varphi_{f}(\rho)=\varphi_{f}^{\prime}(\rho)=$ 0 and $\varphi_{f}^{\prime \prime}(\rho) \neq 0$. In the case of the symmetric simple exclusion in [13] it is obtained an upper bound for the variance of $\Gamma_{t}(f)$ and in [12] it is proved that

$$
\frac{1}{\sqrt{\theta \log (\theta)}} \int_{0}^{\theta t} f\left(\eta_{s}\right) d s \underset{\theta \rightarrow+\infty}{\longrightarrow} \mathcal{B}(C t) .
$$

For symmetric zero-range processes last question is open, but (4) is conjectured to hold for these processes, see [12].

For non zero mean processes, like for example the asymmetric simple exclusion, much less is known. Obviously that, we can get upper bounds on the variance of $\Gamma_{t}(f)$ using the symmetric part of the generator and the results presented above. For the asymmetric simple exclusion, it was proved in [14] that for local functions $f$ such that $\varphi_{f}(\rho)=0$ and $\varphi_{f}^{\prime}(\rho) \neq 0$ or $\varphi_{f}(\rho)=\varphi_{f}^{\prime}(\rho)=0$ and $\varphi_{f}^{\prime \prime}(\rho) \neq 0$, and for $\rho \neq 1 / 2$, the variance of $\Gamma_{t}(f)$ is bounded from above by $C t$, where $C$ is a constant. In a forthcoming paper [1] we are able to obtain sharp bounds in the remaining cases for the asymmetric exclusion and also for asymmetric zero-range processes.

Our approach to these problems is completely different from the ones used in the papers mentioned above. We consider general exclusion processes and by using the local Boltzmann-Gibbs Principle, we are able to relate additive functionals of local functions $f$ with additive functionals of the density of particles. Then, since for those systems, the Central limit Theorem for the density of particles is very well studied, we obtain upper bounds on the variance of $\Gamma_{t}(f)$ and we are able to identify its limit.

This paper is organized as follows. On the second section, we define our microscopic dynamics, namely one-dimensional exclusion type models whose dynamics depends on a local function which is assumed to turn the dynamics elliptic and reversible. On the third section, we recall some results on the scaling limit of the density of particles and we state that the additive functional of an Ornstein-Uhlenbeck process evaluated in a proper indicator function, converges and we identify its limit $z_{t}$ as a fractional Brownian motion with Hurst exponent $H=3 / 4$. On the fourth section, we state that for local functions $f$ such that $\varphi_{f}(\rho)=0$ and $\varphi_{f}^{\prime}(\rho) \neq 0, n^{-3 / 2} \Gamma_{t n^{2}}(f)$ converges 
as $n \rightarrow+\infty$ to $\varphi_{f}^{\prime}(\rho) z_{t}$. The fifth section is devoted to the sketch of the proof of the Local Boltzmann-Gibbs Principle and on the sixth section we present some examples and we discuss the case of symmetric/asymmetric jump rates.

\section{Exclusion processes}

In this section we describe our microscopic dynamics. Let $\left\{\eta_{t}: t \geq 0\right\}$ be a Markov process with space state $\Omega:=\{0,1\}^{\mathbb{Z}}$. The occupation variables are defined in such a way that for $x \in \mathbb{Z}, \eta(x)=1$ if the site $x$ is occupied, otherwise $\eta(x)=0$. At each site $x \in \mathbb{Z}$, there exists a random time clock, with exponential distribution with parameter 1 . If the clock rings at the site $x$, either there is no particle at that site and one has to wait a new random time, or there is a particle at that site and it jumps according to some rate function that we define as follows. Let $r: \Omega \rightarrow \mathbb{R}$ be a local function that satisfies:

\section{(Ellipticity)}

i) There exists $\varepsilon_{0}>0$ such that $\varepsilon_{0}<r(\eta)<\varepsilon_{0}^{-1}$ for any $\eta \in \Omega$.

ii) For any $\eta, \xi \in \Omega$, such that $\eta(x)=\xi(x)$ for $x \neq 0,1$, then $r(\eta)=r(\xi)$. (Reversibility)

The dynamics can be formally described by means of a generator, which is given on local functions $f: \Omega \rightarrow \mathbb{R}$ by:

$$
\mathcal{L} f(\eta)=\sum_{x \in \mathbb{Z}} r\left(\tau_{x} \eta\right)\left(f\left(\eta^{x, x+1}\right)-f(\eta)\right)
$$

where

$$
\eta^{x, y}(z)= \begin{cases}\eta(y), & z=x \\ \eta(x), & z=y \\ \eta(z), & z \neq x, y\end{cases}
$$

and $\tau_{x}$ is the space translation by $x$, namely, for $y \in \mathbb{Z} \tau_{x} \eta(y):=\eta(x+y)$.

The invariant measures for these processes are $\left\{\nu_{\rho}: \rho \in[0,1]\right\}$, where for $\rho \in[0,1], \nu_{\rho}$ denotes the Bernoulli product measure of constant parameter $\rho$. Under this measure the occupation variables $\{\eta(x): x \in \mathbb{Z}\}$ are independent and $\nu_{\rho}(\eta: \eta(x)=1)=\rho$. Here and in the sequel, for $T>0$, we denote by $\mathcal{D}([0, T], \Omega)(\mathcal{C}([0, T], \Omega))$ the space of càdlàg (continuous) trajectories from $[0, T)$ to $\Omega$. We denote by $\mathbb{E}_{\rho}$ the expectation with respect to $\mathbb{P}_{\rho}$ - the distribution of $\left\{\eta_{t}: t \geq 0\right\}$ in the space $\mathcal{D}([0, T], \Omega)$ starting from $\nu_{\rho}$. 


\section{Scaling Limits of the density of particles}

As mentioned in the introduction, our approach is to related the additive functional of local functions $f$ such that $\varphi_{f}(\rho)=0$ and $\varphi_{f}^{\prime}(\rho) \neq 0$, with the additive functional of the density of particles. Then, by using the known results on the scaling limits of the density of particles we are able to deduce the corresponding scaling limits for the additive functional of $f$. We start by recalling the results that concern the density of particles for the exclusion processes that we have defined above.

\subsection{Hydrodynamic Limit}

For each configuration $\eta$ we denote by $\pi^{n}(\eta ; d u)$ the empirical measure given by:

$$
\pi^{n}(\eta ; d u)=\frac{1}{n} \sum_{x \in \mathbb{Z}} \eta(x) \delta_{x / n}
$$

where $\delta_{x / n}$ is the Dirac measure at $x / n$ and $\pi_{t}^{n}(\eta, d u):=\pi^{n}\left(\eta_{t}, d u\right)$.

Under a diffusive scaling of time $t n^{2}$ and for a set of initial meaures associated to a sufficiently smooth profile, the hydrodynamic limit for $\left\{\eta_{t}: t \geq 0\right\}$ was obtained by [5]. The hydrodynamic limit is a Law of Large Numbers for the empirical measure in the following sense. Fix an initial profile sufficiently smooth $\gamma: \mathbb{R} \rightarrow[0,1]$. If for an initial distribution $\left\{\mu_{n}: n \geq 1\right\}$ $\left(\eta_{0} \sim \mu_{n}\right)$ associated to the profile $\gamma(\cdot)$, the empirical measure at time $t=0$ converges to the deterministic measure $\gamma(u) d u$, then for any time $t>0$, the empirical measure at time $t$ converges to the deterministic measure $\rho(t, u) d u$, where $\rho(t, u)$ is the unique weak solution of the corresponding hydrodynamic equation with initial condition $\gamma(\cdot)$.

\subsection{Equilibrium fluctuations}

Now we recall the Central Limit Theorem for the empirical measure for the exclusion processes described above and starting from the invariant state $\nu_{\rho}$. Let $\mathcal{S}(\mathbb{R})$ denote the Schwarz space of test functions and let $\mathcal{S}^{\prime}(\mathbb{R})$ be its dual. For $g \in \mathcal{S}(\mathbb{R})$, the density fluctuation field is defined as

$$
y_{t}^{n}(g):=\frac{1}{\sqrt{n}} \sum_{x \in \mathbb{Z}} g\left(\frac{x}{n}\right)\left\{\eta_{t n^{2}}(x)-\rho\right\} .
$$

It was proved in $[3]$ that $\left\{y_{t}^{n}: t \in[0, T]\right\}$ converges in distribution with respect to the Skorohod topology of $\mathcal{D}\left([0, T], \mathcal{S}^{\prime}(\mathbb{R})\right)$ to the stationary solution 
of the Ornstein-Uhlenbeck equation

$$
d y_{t}=D(\rho) \Delta y_{t} d t+\sqrt{2 D(\rho) \rho(1-\rho)} \nabla d \mathcal{B}_{t},
$$

where $\mathcal{B}_{t}$ is a $\mathcal{S}^{\prime}(\mathbb{R})$-valued Brownian motion and $D(\rho)$ is the diffusion coefficient. In particular, this means that the trajectories of the limit field $y_{t}$ are in $\mathcal{C}\left([0, T], \mathcal{S}^{\prime}(\mathbb{R})\right)$ and that $y_{0}$ is a white noise of variance $\rho(1-\rho)$ namely for any $g \in \mathcal{S}(\mathbb{R})$, the real-valued random variable $y_{0}(g)$ has a normal distribution of mean zero and variance $\rho(1-\rho) \int(g(x))^{2} d x$.

Now, we state a fundamental result in which we state the convergence of the additive functional of $y_{t}$ solution of (7):

Theorem 2. Fix a stationary solution $\left\{y_{t}: t \in[0, T]\right\}$ of (7). For $x \in \mathbb{R}$, let $i_{\varepsilon}(x): y \mapsto \varepsilon^{-1} 1_{(0,1]}\left((y-x) \varepsilon^{-1}\right)$. For each $\varepsilon \in(0,1)$, let $\left\{z_{t}^{\varepsilon}: t \in[0, T]\right\}$ be defined as

$$
z_{t}^{\varepsilon}=\int_{0}^{t} y_{s}\left(i_{\varepsilon}\right) d s .
$$

Then, the process $\left\{Z_{t}^{\varepsilon}: t \in[0, T]\right\}$ converges in distribution with respect to the uniform topology of $\mathrm{C}([0, T], \mathbb{R})$, as $\varepsilon \rightarrow 0$, to a fractional Brownian motion $\left\{z_{t}: t \in[0, T]\right\}$ of Hurst exponent $H=3 / 4$.

\section{Additive functionals}

As mentioned in the introduction, our goal consists in obtaining functional limit theorems for observables of the processes $\left\{\eta_{t}: t \geq 0\right\}$ as defined in section 2. For these processes it holds that:

Theorem 3. For a local function $f: \Omega \rightarrow \mathbb{R}$, the process $\left\{\Gamma_{t n^{2}}(f): t \in\right.$ $[0, T]\}$ defined as

$$
\Gamma_{t n^{2}}(f)=\frac{1}{n^{3 / 2}} \int_{0}^{t n^{2}}\left(f\left(\eta_{s}\right)-\varphi_{f}(\rho)\right) d s
$$

converges in distribution with respect to the uniform topology of $\mathrm{C}([0, T], \mathbb{R})$ to $\left\{\varphi_{f}^{\prime}(\rho) z_{t}: t \in[0, T]\right\}$, where $\left\{z_{t}: t \in[0, T]\right\}$ is the same as in Theorem 2 .

The proof of this result is a consequence of the local Boltzmann-Gibbs Principle whose proof is sketched in the next section. 


\section{Proof of the Local Boltzmann-Gibbs Principle}

The proof of the local Boltzmann-Gibbs Principle as stated in Theorem 1 is divided into four steps. The main ingredients that we use are the KipnisVaradhan inequality (see [10]) and the spectral gap inequality (see [11]).

1. Firstly, we compare the additive functional of $f$ with the additive functional of $\psi_{f}(\ell):=E_{\rho}\left[f \mid \sum_{x=1}^{\ell} \eta(x)\right]$, using the:

\section{Lemma 1 (One-block estimate).}

Let $f: \Omega \rightarrow \mathbb{R}$ be a local function such that $\varphi_{f}(\rho)=0$. Then, there exists $c=c(f, \rho)$ such that for any $\ell \geq k$ and any $t \geq 0$ :

$$
\mathbb{E}_{\rho}\left[\left(\int_{0}^{t}\left\{f\left(\eta_{s}\right)-\psi_{f}\left(\ell ; \eta_{s}\right)\right\} d s\right)^{2}\right] \leq c t \ell^{2} \operatorname{Var}\left(f ; \nu_{\rho}\right),
$$

where $\operatorname{Var}\left(f ; \nu_{\rho}\right)$ denotes the variance of $f$ with respect to $\nu_{\rho}$.

2. Secondly, we compare the additive functional of $\psi_{f}(\ell)$ with the additive functional of $\psi_{f}(2 \ell)$, using the:

\section{Lemma 2 (Renormalization step).}

Let $f: \Omega \rightarrow \mathbb{R}$ be a local function such that $\varphi_{f}(\rho)=0$. There exists $c=c(f, \rho)$ such that for any $\ell \geq k$ and any $t \geq 0$ :

$$
\mathbb{E}_{\rho}\left[\left(\int_{0}^{t}\left\{\psi_{f}\left(\ell ; \eta_{s}\right)-\psi_{f}\left(2 \ell ; \eta_{s}\right)\right\} d s\right)^{2}\right] \leq\left\{\begin{array}{l}
c t \ell, \text { if } \varphi_{f}^{\prime}(\rho) \neq 0, \\
c t, \text { if } \varphi_{f}^{\prime}(\rho)=0 .
\end{array}\right.
$$

3. Thirdly, we compare the additive functional of $\psi_{f}(\ell)$ with the additive functional of $\psi_{f}\left(2^{m} \ell\right)$, using the renormalization step $m$ times.

\section{Lemma 3 (Two-blocks estimate).}

Let $f: \Omega \rightarrow \mathbb{R}$ be a local function such that $\varphi_{f}(\rho)=0$. Then, there exists $c=c(f, \rho)$ such that for any $\ell \geq k$ and any $t \geq 0$ :

$$
\mathbb{E}_{\rho}\left[\left(\int_{0}^{t} \psi_{f}\left(k ; \eta_{s}\right)-\psi_{f}\left(\ell ; \eta_{s}\right) d s\right)^{2}\right] \leq\left\{\begin{array}{l}
c t \ell, \text { if } \varphi_{f}^{\prime}(\rho) \neq 0, \\
c t(\log \ell)^{2}, \text { if } \varphi_{f}^{\prime}(\rho)=0 .
\end{array}\right.
$$

4. Finally, we replace $\psi_{f}\left(2^{m} \ell\right)$ by the corresponding function of $\eta^{2^{m} \ell}$ using the:

\section{Proposition 1 (Equivalence of Ensembles).}

Let $f: \Omega \rightarrow \mathbb{R}$ be a local function. Then there exists a constant $c=c(f, \rho)$ such that for any $\ell \geq k$ :

$$
\int\left(\psi_{f}(\ell, \eta)-\varphi_{f}^{\prime}(\rho)\left(\eta^{\ell}-\rho\right)-\frac{\varphi_{f}^{\prime \prime}(\rho)}{2}\left(\left(\eta_{s}^{\ell}-\rho\right)^{2}-\frac{\rho(1-\rho)}{\ell}\right)\right)^{2} d \nu_{\rho} \leq \frac{c}{\ell^{3}} .
$$




\section{Examples}

In this section we present some examples for which we can derive the precise statement of the theorems given above. We start by the mean-zero exclusion process.

\subsection{Mean-Zero Exclusion}

The mean-zero exclusion process is defined as in section 2 , but in this case after an exponential time of parameter 1 , a particle at the site $x$ jumps to the site $x+y$ with probability $p(y)$. We assume the following conditions on the probability measure $p: \mathbb{Z} \backslash\{0\} \rightarrow[0,1]$ :

1) $p(\cdot)$ has finite range, that is, there exists $M>0$ such that $p(z)=0$ whenever $|z|>M$;

2) $p(\cdot)$ is irreducible, i.e. $\mathbb{Z}=\operatorname{span}\{z \in \mathbb{Z} ; p(z)>0\}$;

3) $p(\cdot)$ has mean-zero: $\sum_{z \in \mathbb{Z}} z p(z)=0$.

Example: If we take $p(1)=2 / 3, p(-2)=1 / 3$ and $p(z)=0$ if $z \neq-2,1$, then the process is an example of an asymmetric mean-zero exclusion.

We define the Markov process $\left\{\eta_{t}^{\text {ex }}: t \geq 0\right\}$, whose generator acts over local functions $f: \Omega \rightarrow \mathbb{R}$ as

$$
\mathcal{L}_{\mathrm{ex}} f(\eta)=\sum_{x, y \in \mathbb{Z}} p(y) \eta(x)(1-\eta(x+y))\left(f\left(\eta^{x, x+y}\right)-f(\eta)\right),
$$

with $p(\cdot)$ satisfying 1$), 2)$ and 3$)$ and $\eta^{x, x+y}$ as in (5). The measures $\left\{\nu_{\rho}: \rho \in\right.$ $[0,1]\}$ are invariant, but they are not necessarily reversible (that is true if and only if $p(\cdot)$ is symmetric). Thus, asymmetric mean-zero exclusion processes are diffusive and non-reversible systems. We can define the density fluctuation field $\left\{y_{t}^{n}: t \in[0, T]\right\}$ as in (6) and we have that:

Proposition 2. The process $\left\{y_{t}^{n}: t \in[0, T]\right\}$ converges in distribution with respect to the Skorohod topology of $\mathcal{D}\left([0, T], \mathcal{S}^{\prime}(\mathbb{R})\right)$ to the stationary solution of the Ornstein-Uhlenbeck equation

$$
d y_{t}=D(\rho) \Delta y_{t} d t+\sqrt{2 D(\rho) \rho(1-\rho)(\rho)} \nabla d \mathcal{B}_{t},
$$

where $D(\rho)$ is the diffusion coefficient.

The results presented above allow us to get the scaling limits of additive functionals as in Theorem 3. We notice that in spite of having stated the theorem for reversible systems (see condition ii) on $r$ ), we can prove the local Boltzmann-Gibbs Principle for non-reversible systems, since the KipnisVaradhan inequality also fits these systems, see [4] for details. 


\subsection{Symmetric simple exclusion}

Consider $\mathcal{L}_{e x}$ as above with $p(\cdot)$ such that $p(1)=p(-1)=1 / 2$ and $p(z)=0$ for $z \neq-1,1$. We notice that for this process the measures $\left\{\nu_{\rho}: \rho \in[0,1]\right\}$ are invariant and reversible. In this case we have that:

Proposition 3. The process $\left\{y_{t}^{n}: t \in[0, T]\right\}$ converges in distribution with respect to the Skorohod topology of $\mathcal{D}\left([0, T], \mathcal{S}^{\prime}(\mathbb{R})\right)$ to the stationary solution of the Ornstein-Uhlenbeck equation

$$
d y_{t}=\frac{1}{2} \Delta y_{t} d t+\sqrt{\rho(1-\rho)} \nabla d \mathcal{B}_{t}
$$

The results presented above allow us to get the scaling limits of additive functionals as stated in Theorem 3 .

\subsection{The weakly asymmetric simple exclusion}

Now, we introduce an exclusion type process which has a drift towards the right. For that purpose, take $\mathcal{L}_{e x}$ as above with $p(\cdot)$ given by $p_{n}(1)=\frac{1}{2}+\frac{a_{n}}{2}$, $p_{n}(-1)=\frac{1}{2}-\frac{a_{n}}{2}$ and $p_{n}(z)=0$ if $z \neq-1,1$. The measures $\left\{\nu_{\rho}: \rho \in[0,1]\right\}$ are invariant but not reversible.

\subsubsection{The hydrodynamic scaling}

If $a_{n}:=\frac{1}{n}$, then we have that

Proposition 4. The process $\left\{y_{t}^{n}: t \in[0, T]\right\}$ converges in distribution with respect to the Skorohod topology of $\mathcal{D}\left([0, T], \mathcal{S}^{\prime}(\mathbb{R})\right)$ to the stationary solution of the Ornstein-Uhlenbeck equation

$$
d y_{t}=\frac{1}{2} \Delta y_{t} d t+(1-2 \rho) \nabla y_{t} d t+\sqrt{\rho(1-\rho)} \nabla d \mathcal{B}_{t}
$$

In this case the Ornstein-Uhlenbeck process has a drift, nevertheless one can get the same result as stated in Theorem 3 .

\subsubsection{The KPZ scaling}

Fix a density $\rho=1 / 2$. Then, inserting this in the previous stochastic partial differential equation, we can see that the limit field is the same as in the symmetric simple exclusion (so a weak asymmetry does not have influence!), 
see [8]. In this case the "correct" strength asymmetry is $a_{n}=1 / \sqrt{n}$. In this case we have

Proposition 5. The process $\left\{y_{t}^{n}: t \in[0, T]\right\}$ converges in distribution with respect to the Skorohod topology of $\mathcal{D}\left([0, T], \mathcal{S}^{\prime}(\mathbb{R})\right)$ to the stationary solution of the stochastic Burgers equation:

$$
d y_{t}=\frac{1}{2} \Delta y_{t} d t+\left(\nabla y_{t}\right)^{2} d t+\sqrt{\rho(1-\rho)} \nabla d \mathcal{B}_{t}
$$

In this case, the limit density field is no longer an Ornstein-Uhlenbeck process, so Theorem 2 is not useful in this case. Nevertheless, for $y_{t}$ solution of (9) we can also prove that:

Theorem 4. Let $\left\{y_{t}: t \in[0, T]\right\}$ be a stationary solution of (9). For $\varepsilon>0$, let $\tilde{z}_{t}^{\varepsilon}=\int_{0}^{t} y_{s}\left(i_{\varepsilon}\right) d s$. Then there exists $\left\{\tilde{z}_{t}: t \in[0, T]\right\}$ such that, $\left\{\tilde{z}_{t}^{\varepsilon}\right.$ : $t \in[0, T]\}$ converges in distribution with respect to the uniform topology of $\mathcal{C}([0, T], \mathbb{R})$, as $\varepsilon \rightarrow 0$, to $\left\{\tilde{z}_{t}: t \in[0, T]\right\}$.

And as a consequence we have that

Theorem 5. Let $f: \Omega \rightarrow \mathbb{R}$ be a local function such that $\varphi_{f}(1 / 2)=0$. Then, $\left\{\Gamma_{t n^{2}}(f): t \in[0, T]\right\}$ as defined in (8) converges in distribution with respect to the uniform topology of $\mathcal{C}([0, T], \mathbb{R})$ to $\left\{\varphi_{f}^{\prime}(1 / 2) \tilde{z}_{t}: t \in[0, T]\right\}$, where $\tilde{z}_{t}$ is the same as in Theorem 4.

\subsection{Symmetric simple exclusion/Asymmetric simple exclusion}

Here we discuss the differences between the bounds on the variance of additive functionals of the symmetric simple exclusion (ssep) and the asymmetric simple exclusion process (asep), both defined on $\mathbb{Z}$. The latter process is defined through $\mathcal{L}_{e x}$ as above, but with $p(1):=p, p(-1):=1-p$ with $p \neq 1 / 2$ and $p(z)=0$ for $z \neq-1,1$.

Let $f$ be a local function.

1) If $\varphi_{f}(\rho)=0$ and $\varphi_{f}^{\prime}(\rho) \neq 0$, then:

$$
\operatorname{Var}\left(\Gamma_{t}(f) ; \nu_{\rho}\right) \leq C t^{3 / 2} \text { in ssep }
$$

and

$$
\operatorname{Var}\left(\Gamma_{t}(f) ; \nu_{\rho}\right) \leq\left\{\begin{array}{ll}
C t^{4 / 3}, & \rho=\frac{1}{2} \\
C t, & \rho \neq \frac{1}{2}
\end{array}\right. \text { in asep. }
$$

With the results presented above one gets the correct upper bound for the ssep. The method presented above does not give the correct upper bound in 
the asep. In $[1,14]$ it is proved the correct bound in the asep when $\rho \neq 1 / 2$ and in [1] the upper bound $t^{3 / 2}$ is obtained when $\rho=1 / 2$. The correct upper bound when $\rho=1 / 2$ is still out of reach.

2) If $\varphi_{f}(\rho)=\varphi_{f}^{\prime}(\rho)=0, \varphi_{f}^{\prime \prime}(\rho) \neq 0$, then:

$$
\operatorname{Var}\left(\Gamma_{t}(f) ; \nu_{\rho}\right) \leq C t \log (t) \text { in ssep }
$$

and

$$
\operatorname{Var}\left(\Gamma_{t}(f) ; \nu_{\rho}\right) \leq C t \text { in asep. }
$$

With the results presented above one gets the upper bound $C t(\log (t))^{2}$ in the ssep. The correct upper bound was obtained in [12]. In the asep, in [14] ([1]) the correct upper bound was obtained for $\rho \neq 1 / 2(\rho=1 / 2)$.

3) If $\varphi_{f}(\rho)=\varphi_{f}^{\prime}(\rho)=\varphi_{f}^{\prime \prime}(\rho)=0, \varphi_{f}^{\prime \prime \prime}(\rho) \neq 0$, then $\operatorname{Var}\left(\Gamma_{t}(f) ; \nu_{\rho}\right) \leq C t$ for both ssep and asep.

This bound was firstly obtained in [15] and with the results presented above we can also get the correct upper bound in these cases. Above $C$ is a constant.

\section{References}

1. Bernardin, C.; Gonçalves, P.; Jara, M.; Sethuraman, S. (2012): Additive functionals of finite range exclusion and zero-range processes, in preparation.

2. Brox, T. and Rost, H. (1984): Equilibrium fluctuations of stochastic particle systems: the role of conserved quantities, Annals of Probability, 12 (3), 742-759.

3. Chang, C. (1994): C Equilibrium fluctuations of gradient reversible particle systems, Probability Theory and Related Fields, 100 (3), 269-283.

4. Chang, C.; Landim, C. and Olla, S. (2001): Equilibrium fluctuations of asymmetric simple exclusion processes in dimension $d \geq 3$, Probability Theory and Related Fields, 119 (3), 381-409.

5. Funaki, T.; Uchiyama, K. and Yau, Y. (1996): Hydrodynamic limit for lattice gas reversible under Bernoulli measures. Nonlinear stochastic PDE's, Springer.

6. Gonçalves, P. (2008): Central Limit Theorem for a Tagged Particle in Asymmetric Simple Exclusion, Stochastic Process and their Applications, 118, 474-502.

7. Gonçalves, P. and Jara, M. (2011): Universality of KPZ equation, available online at arXiv: 1003.4478.

8. Gonçalves, P and Jara, M. (2011): Crossover to the KPZ equation, accepted for publication in the Annales Henri Poincaré.

9. Gonçalves, P. and Jara, M. (2011): Scaling limits of additive functionals of interacting particle systems, available online at arXiv:1103.3722 and submitted.

10. Kipnis, C. and Varadhan, S. (1986): Central limit theorem for additive functionals of reversible Markov processes and applications to simple exclusions, Communications on Mathematical Physics, 104 (1), 1-19.

11. Quastel, J. (1992): Diffusion of color in the simple exclusion process, Comm. Pure Appl. Math., 45 (6), 623-679. 
12. Quastel, J.; Jankowski, H.; Sheriff, J. (2002): Central limit theorem for zero-range processes, Special issue dedicated to Daniel W. Stroock and Srinivasa S. R. Varadhan on the occasion of their 60th birthday, Methods Appl. Anal., 9 (3), 393-406.

13. Sethuraman, S. (2000): Central Limit Theorems for Additive Functionals of the Simple Exclusion Process, Annals of Probability, 28, 277-302.

14. Seppäläinen, T. and Sethuraman S. (2003): Transience of second-class particles and diffusive bounds for additive functionals in one-dimensional asymmetric and exclusion processes, Annals of Probability, 31 (1), 148-169.

15. S. Sethuraman, L. Xu. (1996): A central limit theorem for reversible exclusion and zero-range particle systems, Annals of Probability, 24 (4), 1842-1870.

16. Varadhan, S. (1995): Self-diffusion of a tagged particle in equilibrium for asymmetric mean zero random walk with simple exclusion, Annales de l'Institut Henri Poincaré, 31, 273-285. 